\title{
DOENÇAS SEXUALMENTE TRANSMISSÍVEIS: A PERCEPÇÃO DOS ADOLESCENTES DE UMA ESCOLA PÚBLICA
}

Fabrine Aguilar Jardim¹, Thamara de Souza Campos², Renan Neves da Mata ${ }^{3}$, Maria da Penha Rodrigues Firmes ${ }^{4}$

\begin{abstract}
RESUMO: Este estudo descritivo de abordagem qualitativa buscou conhecer a percepção de adolescentes sobre doenças sexualmente transmissíveis. Foram entrevistados 30 adolescentes de uma escola pública de Diamantina-Minas Gerais, entre agostoe dezembro de 2012. As entrevistas transcritas sofreram o processo de identificação dos núcleos de sentidos e categorização pela Análise de Conteúdo o originaramse duas categorias: Conhecimento sobre doenças sexualmente transmissiveis e Formas de aquisição de informações sobre doenças sexualmente transmissiviveis. A camisinha representou a principal forma de prevenção, os riscos ocasionados à saúde e vida social forammorte, aids e preconceito. Os adolescentes conversam com amigos/colegas e as ações sobre essas doenças foram vivenciadas por meio das aulase palestras. A percepção dos adolescentes sobre doenças sexualmente transmissíveis contribui para repensar a importância de projetos, novos estudos e políticas públicas para reduzir vulnerabilidade e comportamentos de riscos durante a vivência da sexualidade na adolescência. DESCRITORES: Doenças sexualmente transmissíveis; Adolescente; Pesquisa qualitativa.

\section{SEXUALLY TRANSMITTED DISEASES: THE PERCEPTION OF ADOLESCENTS IN A STATE SCHOOL}

ABSTRACT: This descriptive study with a qualitative approach sought to investigate the adolescents' perception regarding sexually transmitted diseases. Interviews were held with 30 adolescents from a state school in Diamantina, Minas Gerais, between August and December 2012. The interviews were transcribed and underwent the process of identification of the nuclei of meaning and categorization by Content Analysis, two categories emerging: Knowledge of sexually transmitted diseases and Ways of acquiring information on sexually transmitted diseases. The condom represented the main form of prevention, and the risks caused to healt and social life were death, AIDS and prejudice. The adolescents converse with friends/class-mates, and the actions in relation to these diseases were experienced through lessons and presentations. The adolescents' perception regarding sexually transmitted diseases contributes to re-thinking the importance of projects, further studies and public policies for reducing vulnerability and behaviors of risk during the experience of sexuality in adolescence. DESCRIPTORS: Sexually transmitted diseases; Adolescent; Qualitative research.

\section{ENFERMEDADES SEXUALMENTE TRANSMISIBLES: LA PERCEPCIÓN DE LOS ADOLESCENTES DE UNA ESCUELA PÚBLICA}

RESUMEN: Este estudio descriptivo de abordaje cualitativo cuyo objetivo fue conocer la percepción de adolescentes sobre enfermedades sexualmente transmisibles. Fueron entrevistados 30 adolescentes de una escuela pública de Diamantina - Minas Gerais, entre agosto y diciembre de 2012. Las entrevistas transcriptas pasaron por proceso de identificación de los núcleos de sentidos y categorización por el Análisis de Contenido y resultaron en dos categorías: Conocimiento acerca de enfermedades sexualmente transmisibles y Formas de adquisición de informaciones sobre enfermedades sexualmente transmisibles. El preservativo fue referido como la principal forma de prevención, los riesgos a la salud y la vida social fueron muerte, SIDA y prejuicio. Los adolescentes charlaron con amigos/ compañeros y las acciones acerca de esas enfermedades fueron vividas por medio de las clases y conferencias. La percepción de los adolescentes sobre enfermedades sexualmente transmisibles contribuye para repensar la importancia de proyectos, nuevos estudios y políticas públicas para reducir vulnerabilidad y comportamientos de riesgos durante la vivencia de la sexualidad en la adolescencia. DESCRIPTORES: Enfermedades sexualmente transmisibles; Adolescente; Investigación cualitativa.

\footnotetext{
*Artigo desenvolvido a partir de Trabalho de Conclusão de Curso de Graduação em Enfermagem apresentado à Universidade Federal dos Vales do Jequitinhonha e Mucuri - UFVJM, em 2013.

${ }^{1}$ Enfermeira.

${ }^{2}$ Enfermeira da Saúde da Família no Município de Diamantina. Mestre em Ciências da Saúde: Saúde da Criança e do Adolescente. Preceptora de projetos no Pró-Saúde Enfermagem UFVJM.

${ }^{3}$ Enfermeiro Residente em Enfermagem na Atenção Básica/Saúde da Família da Universidade Federal de São João del-Rei.

${ }^{4}$ Enfermeira. Doutora em Enfermagem. Professora do Departamento de Enfermagem da UFVJM e Líder do Grupo Vida - Grupo de Pesquisas em Violência em Diamantina e Arredores, com ênfase em Violência Doméstica e Alcoolismo.
} 


\section{INTRODUÇÃO}

A adolescência é um período de transição entre a infância e a vida adulta, caracterizado por dúvidas e sentimentos conflituosos em relação à vivência da sexualidade $^{(1)}$. Os adolescentes constituem um grupo que requer atenção diferenciada, pois muitos iniciam a vida sexual quando ainda apresentam baixo conhecimento sobre as doenças sexualmente transmissíveis (DST) e percepção equivocada sobre o risco pessoal de adquirir essas doenças, considerando a ausência de práticas efetivas de proteção ${ }^{(2)}$.

O aumento dos índices de DST se deve, também, ao início precoce da atividade sexual, que se torna cada vez mais comum entre os adolescentes. Estes muitas vezes não se encontram preparados para assumir tal responsabilidade, devido à imaturidade, inexperiência e falta de acesso a informações, seja no ambiente escolar ou familiar, pelas características próprias desta fase da $\mathrm{vida}^{(3)}$.

Dados do Ministério da Saúde ${ }^{(4)}$ evidenciam que as DST são vistas como um grave problema de saúde pública por afetarem muitas pessoas e os sinais e sintomas serem de difícil identificação, assim como o acesso ao tratamento correto. Além disso, esses agravos à saúde estão relacionados com práticas sexuais e, portanto, com tabus, desinformação, vergonha, constrangimento e preconceito, dificultando o seu reconhecimento e a busca precoce por assistência qualificada.

Para a Organização Mundial de Saúde ${ }^{(5)}$, as DST de maior recorrência no Brasil, na população sexualmente ativa, somam a cada ano 937.000 casos de sífilis; 1.541 .800 casos de gonorréia; 1.967 .200 casos de clamídia; 640.900 casos de herpes genital e 685.400 casos de Papilomavírus Humano (HPV). Além disso, o número de soropositivos registrados pelo MS chegou a 592.914 desde a década de 1980 até 2010.

A preocupação com a saúde sexual e reprodutiva dos adolescentes tem atraído atenção especial de profissionais de diversas áreas do conhecimento, dentre elas a enfermagem, despertando interesse pelo seu estudo, a partir de uma perspectiva da subjetividade social nela implicada. Dessa forma, o presente estudo busca identificar a percepção dos adolescentes no que diz respeito ao conhecimento e forma de aquisição de informações sobre as DST, em uma escola pública.

\section{MÉTODO}

Trata-se de um estudo descritivo de abordagem qualitativa, realizado no período de agosto a dezem- bro de 2012, com 30 adolescentes de ambos os sexos, de uma escola estadual da rede pública do município de Diamantina - Minas Gerais. Os participantes da pesquisa foram selecionados por meio de amostragem aleatória e foram critérios de inclusão ter entre $10 \mathrm{e}$ 19 anos de idade e estar regularmente matriculado na escola entre o quinto e o nono ano.

Os dados foram coletados a partir de entrevista semiestruturada, em uma sala reservada na própria escola, por meio de um roteiro de entrevista composto de seis questões discursivas. Foram efetuadas transcrições integrais das entrevistas e os relatos foram submetidos à técnica de análise de conteúdo ${ }^{(6)}$.

A identificação dos participantes se deu por meio de pseudônimos, com intuito de assegurar $\mathrm{o}$ anonimato e o sigilo das informações. Foi apresentada uma lista com nomes de aves brasileiras, na qual os adolescentes escolheram aqueles que possuíam maior afinidade. Isto permitiu de certa forma, um maior envolvimento, liberdade e motivação dos participantes durante a entrevista.

Este estudo foi aprovado pelo Comitê de Ética em Pesquisa da Universidade Federal dos Vales do Jequitinhonha e Mucuri (UFVJM), sob número do protocolo 052/10, atendendo, desta forma, a Resolução n0 196/96, do Conselho Nacional de Saúde. A participação dos adolescentes na pesquisa ocorreu após autorização dos pais ou responsáveis, e assinatura do Termo de Consentimento Livre e Esclarecido (TCLE).

\section{RESULTADOS}

Os 30 adolescentes entrevistados tinham idade mínima de 11 e máxima de 17 anos, sendo a média de idade 13,9 anos. Em relação ao sexo, 21(70\%) eram do sexo feminino e $9(30 \%)$ do sexo masculino.

Com a análise das falas dos adolescentes, emergiram duas categorias, e de cada categoria, duas subcategorias descritas, a seguir:

\section{Categoria I- Conhecimento sobre as doenças sexu- lamente transmissíveis}

Nesta categoria identificaram-se, nos depoimentos, os núcleos de sentidos referentes às formas de prevenção e aos riscos que essas doenças representam para a saúde e vida social.

\section{Subcategoria A: Formas de prevenção}

Ao serem questionados sobre a prevenção das DST, 
os adolescentes relataram que a principal forma consiste no uso de camisinha nas relações sexuais, como observado nas falas:

Métodos contraceptivos, principalmente a camisinha, evitar ficar usando objetos pessoais, como seringas e fazer sexo com segurança e etc. (Maria- Leque)

Ter relação sexual só com camisinha. Só usar materiais descartáveis, até para fazer tatuagem. Não usar seringas que acham. (Águia)

Usar camisinha, tanto seja a masculina ou feminina, não ter uma relação sexual sem a camisinha se não pega doença mesmo. (Bem-te-vi)

Camisinha, comprimidos, pílulas e anticoncepcionais. (Gaivota)

Subcategoria B: Riscos para a saúde e a vida social

Nesta subcategoria as DST foram correlacionadas ao risco que podem trazer tanto para a saúde como para a vida social. Os adolescentes relataram o risco de morte, conforme os depoimentos:

A pessoa vai ficar mais doente, isso atrapalha no sexo e isso no futuro pode causar morte. (Tucano)

Pessoa pode morrer, se tiver um filho pode prejudicar o filho também, outras pessoas que estão ao seu redor. (Garça)

Pode levar a morte. Se não tratar pode espalhar, comprometer as células. A pessoa pode ficar fraca. Ela pode ficar depressiva. (Águia)

Outro aspecto observado foi à associação com a aids, presente nas falas dos adolescentes, de acordo com os exemplos:

Pegar outras doenças, a aids deixa o organismo mais fraco, morte, a aids tem que tomar remédio. (Canário do Campo)

Muitos riscos, tem doenças que não tem cura, aids, por exemplo, não tem cura só tem como prevenir. (Arara)

Saúde piora, transmite aids. (João de Barro)
As falas traduzem também o significado dos riscos que as DST representam para os adolescentes. A aids foi a única doença citada, bem como associação desta com seu aspecto de não possuir cura e com as consequências da mesma para a saúde.

Outros adolescentes citaram ainda o preconceito quando questionados sobre os riscos que essa patologia pode trazer a vida social:

Preconceito. Não pode mais ter relação sexual com liberdade, até tem pessoa que nem sabe que ela tem aids e faz isso para passar as pessoas. (Falcão)

Muita infelicidade, porque muitas pessoas têm preconceito com essas doenças e que algum dia não quero que passe para alguém da minha família. Ter muito preconceito, não pode ter relação normal com o parceiro, não poderá ter filho, até que pode, tem que ter acompanhamento médico. (Ema)

Deficiência física, moral e várias outras coisas, como o bullying, preconceito, rejeição pelos familiares. (Mãe da Lua)

Além da associação com preconceito, as DST foram associadas pelos adolescentes a outras variações de significados à vida social.

\section{Categoria II- Forma de aquisição de informações sobre as doenças sexualmente transmissíveis}

Os adolescentes afirmaram adquirir informações por meio de conversas com familiares, amigos, pais e mães. Eles citaram ações realizadas no ambiente escolar sobre as DST, como palestras, aula de ciências e projetos universitários no município. Desta forma os núcleos de sentido foram organizados em duas subcategorias, apresentadas a seguir:

\section{Subcategoria A: Pessoas com quem conversam}

Conversar sobre as DST na adolescência significa estabelecer um cotidiano de diálogo consolidado no respeito e liberdade. Os participantes relataram ter maior liberdade para falar sobre este assunto com os colegas e amigos, sendo eles da escola ou da rua onde moram. Outros interlocutores também foram citados, como por exemplo, conversas com os professores, com os pais e com os parceiros, como observado nas falas: 
Com os colegas. Não converso com os pais, sei lá. Com a namorada. Tenho mais liberdade de com a namorada do que com os pais. (Tucano)

Ah, com professores, colegas mesmo, de sala. Converso com meu pai e mãe. (Tesourão)

Converso com minha mãe e minhas colegas da escola, com minhas irmãs também converso demais. (Gaivota)

Com meus pais. Eles me chamam pra conversar, eles me chamam, não fico com vergonha, já sou acostumado a conversar com eles. Colegas da rua e da escola. (Saracura)

\section{Subcategoria B: Ações na escola em que estudam}

As falas dos adolescentes apresentaram conteúdos relacionados à forma de aquisição de informações no ambiente escolar, tais como aulas de ciências, em palestras ministradas por profissionais de saúde e por acadêmicos de projetos de universidade:

Sim. Foi este ano na aula de ciências, foi bom, discutimos sobre vários tipos de doenças. Estamos aprendendo mais. (Codorna mineira)

Sim. Foi legal, tirou bastante dúvida. Foi palestra. Homens e mulheres médicos que deram a palestra. (Perdiz)

Sim. No ano passado a estagiária estava dando palestra, mostrando o modo de se prevenir. Veio uma turma falando, no ano passado da faculdade. Ficaram aqui dois dias. (Beija- flor)

Os adolescentes sinalizaram ações educativas com discussão e elucidação de dúvidas, contudo alguns adolescentes responderam que não houve estas atividades educativas sobre as DST na escola.

\section{DISCUSSÃO}

O conhecimento dos adolescentes acerca das DST neste estudo trouxe a perspectiva de que o tema não é totalmente desconhecido por eles. No que diz respeito à subcategoria $\mathrm{A}$, observou-se que a maioria associou de forma correta a prevenção dessas doenças ao uso da camisinha. Em estudo realizado com 746 alunos de duas escolas públicas da cidade do Rio de Janeiro, já no ano de 2003, também houve a presença do termo camisinha como núcleo central nas falas dos adolescentes, sugerindo um processo de incorporação pelos mesmos de conhecimentos relativos à prevenção em saúde reprodutiva e revelando, possivelmente o sucesso das campanhas de educação em saúde, que fazem com que a camisinha seja a principal forma de prevenção conhecida pelos jovens ${ }^{(3)}$.

O uso de métodos preventivos não possui relação direta com o conhecimento dos adolescentes, estes são divulgados, na maioria das vezes, em ações e programas de educação em saúde, mas o seu uso efetivo envolve aspectos históricos e culturais que dificultam uma transformação comportamental para a vivência segura do ato sexual ${ }^{(7)}$. A contracepção de urgência é recomendada para adolescentes como forma de garantia quando há uso inadequado ou o não uso do preservativo, mas isso não previne essas doenças, somente previne a gravidez indesejada ${ }^{(8)}$. Ainda, o baixo nível de informação sobre DST/aids, principalmente, o desconhecimento das formas de infecção é o motivo para a não prevenção desses agravos à saúde entre adolescentes $^{(9)}$.

Quanto à subcategoria $\mathrm{B}$, as DST representaram o sentido de morte, sendo este o núcleo de sentido mais citado, juntamente com variações da forma verbal morrer. A morte, em especial para os jovens é um tabu, os quais pelo curso natural da vida viriam antes a envelhecer. Entretanto, refletir sobre ela é importante, devido ao fato de ter se tornado um problema social entre os adolescentes ${ }^{(10)}$.

A associação dos riscos das DST e aids foi evidenciado em estudo ${ }^{(1)}$ com 180 alunos de uma escola municipal, em Canoas-Rio Grande do Sul. Neste 91,4\% conheciam as DST, sendo a aids a doença mais citada. Esta síndrome se destaca como a mais conhecida pelos adolescentes, que referem-na como uma doença que não tem cura. Algumas doenças sexualmente transmissíveis, com exceção da aids, são pouco conhecidas pelos adolescentes, como exemplo, o HPV e o cancro mole ${ }^{(11)}$.

As preocupações com os riscos para a vida social ficaram evidentes, por meio da repetição do núcleo de sentido preconceito. Para os adolescentes as pessoas possuem preconceito em relação a aids e outras DST por estarem relacionadas à promiscuidade e prostituição. Torna-se relevante a discussão das formas de transmissão das DST com os adolescentes, bem como debater a crença errônea segundo a qual a aparência sinaliza a possibilidade do parceiro ou parceira estar infectado pelo vírus ${ }^{(12)}$. Neste sentido, destaca-se a 
importância da inserção e adequação destes conteúdos, nas ementas curriculares do ensino, com o intuito de trabalhar essa temática no contexto social em que os jovens estão inseridos.

Foi possível perceber que alguns adolescentes não se sentem à vontade ao conversar com seus pais, associando este momento ao sentimento de vergonha, embora a conversa com as mães tenha sido mais citada, quando comparada com os pais. A busca por informações sobre as DST, pelos adolescentes, acontece com os amigos seguidos da família, professores e profissionais de saúde. Entretanto, percebeu que na maioria das vezes, os amigos e pais não estão preparados para responder as demandas dos adolescentes ${ }^{(13)}$.

Nos diálogos sobre as DST o provedor de informações deve ter cuidado e sensibilidade ao tratar sobre elas com os adolescentes, por ser uma temática que desperta emoções e sentimentos como medo, raiva, vergonha, rejeição e negação ${ }^{(14)}$. Outro ponto é que os núcleos familiares desestruturados são menos resilientes e, consequentemente, influenciam na vulnerabilidade para a contração de $\operatorname{DST}^{(13)}$.

A dinâmica da sociedade contemporânea, as suas mudanças presentes nas relações no âmbito das famílias, da escola e trabalho, traz repercussões importantes na formação dos adolescentes. A vulnerabilidade e os riscos físicos, emocionais e sociais a que se expõem, incluem questões como a prática sexual não segura com exposição à infecção por DST/Aids ${ }^{(15)}$.

A escola se configura como um local estratégico para a inserção de ações educativas em saúde, nela os adolescentes devem ser estimulados a participarem de projetos que orientem a respeito da saúde sexual e reprodutiva. Este público necessita de informações para desenvolver um comportamento responsável ${ }^{(14)}$ assumindo o protagonismo e o empoderamento, no que diz respeito ao exercício da sexualidade consciente. $\mathrm{O}$ ambiente escolar configura-se como um cenário de formação, sendo espaço associado com as inter-relações sociais dos adolescentes.

A gestão administrativa das escolas muitas vezes solicita palestras sobre sexualidade, prevenção da gravidez na adolescência, DST e outras que são oferecidas pelos serviços de saúde. Mas a palestra por si só tem pouco valor, por ser uma ação pontual, embora possa servir como contato com a comunidade, diminuir dúvidas e encurtar o caminho até os serviços de saúde. A inserção da universidade nas escolas, em programas de longa duração, nas cidades que contam com esse apoio é uma estratégia que deve ser explorada.
Torna-se fundamental a implementação do Programa Saúde na Escola, o qual prevê a realização de diversas ações conjunta das equipes de saúde e de educação com o objetivo de garantir atenção à saúde e educação integral para os estudantes da rede básica de ensino, buscando intervir preventivamente neste contexto, uma vez que os adolescentes assumem no campo social um papel que pode representar risco a sua saúde durante a exercício da sexualidade ${ }^{(16-17)}$.

\section{CONSIDERAÇÕES FINAIS}

Este estudo possibilitou conhecer o pensar do adolescente sobre as doenças sexualmente transmissíveis, os resultados mostram que a maioria dos sujeitos referiu a camisinha como forma de prevenção destas doenças. A morte, a aids e o preconceito representaram para os adolescentes os principais riscos ocasionados a saúde e a vida social pelas DST. No que diz respeito à aquisição de informações ficou claro que os adolescentes sentem maior liberdade em conversar com amigos e colegas sobre essas doenças e que o ambiente escolar foi considerado um importante cenário para a realização de ações educativas em aulas de ciências, palestras de profissionais de saúde e de acadêmicos do município.

Em função da iniciação da vida sexual precoce dos adolescentes torna-se necessário a disseminação de informações sobre essas doenças, tendo como alvo a prevenção. Por ser a adolescência uma fase de descobertas, dúvidas e curiosidades em relação ao sexo se configuram como um período no qual é importante intervir e orientar. Nada melhor do que incluir essa função no cotidiano do adolescente na sua dimensão familiar, escolar, e social.

A percepção dos adolescentes sobre as DST contribui para repensar a importância de projetos de extensão, novos estudos e reformulação de políticas públicas sobre a temática, com intuito de reduzir a vulnerabilidade e comportamentos de riscos eminentes durante a vivência da sexualidade na adolescência.

\section{REFERÊNCIAS}

1. Theobald VD, Nader SS, Pereira DN, Gerhardt CR, Oliveira FJM. A universidade inserida na comunidade: conhecimentos, atitudes e comportamentos de adolescentes de uma escola pública frente a doenças sexualmente transmissíveis. Rev. AMRIGS. [Internet]. 2012;6(1) [acesso em 03 fev 2013]. Disponível: http://www. amrigs.com.br/revista/56-1/0000095572-6_929.pdf 
2. Doreto DT, Vieira EM. O conhecimento sobre doenças sexualmente transmissíveis entre adolescentes de baixa renda em Ribeirão Preto, São Paulo, Brasil. Cad. Saúde Pública. [Internet] 2007;23(10) [acesso em 01 mar 2013]. Disponível: http://www.scielo.br/pdf/csp/ v23n10/26.pdf

3. Oliveira DC, Gomes AMT, Pontes APM, Salgado LPP. Atitudes, sentimentos e imagens na representação social da sexualidade entre adolescentes. Esc. Anna Nery. [Internet] 2009;13(4) [acesso em 17 fev 2013]. Disponível: http://www.scielo.br/pdf/ean/v13n4/v13n4a18.pdf

4. Ministério da Saúde (BR). A saúde dos adolescentes e jovens. [Internet] [acesso em 17 fev 2013]. Disponível: portal.saude.gov.br/portal/arquivos/multimedia/ adolescente/principal.htm

5. Ministério da Saúde (BR). Saúde da mulher. [Internet] [acesso em 20 fev 2013]. Disponível: http://www.brasil. gov.br/sobre/saude/saude-da-mulher/hiv-e-dst-emmulheres

6. Bardin L. Análise do conteúdo. São Paulo: Edições 70; 2011.

7. Camargo EAI, Ferrari RAP. Adolescentes: conhecimentos sobre sexualidade antes e após a participação em oficinas de prevenção. Ciênc. saúde colet. [Internet] 2009;14(3) [acesso em 01 mar 2013]. Disponível: http://www.scielo.br/pdf/csc/v14n3/30.pdf

8. Rodrigues, MF, Jardim, DP. Conhecimento e uso de contracepção de emergência na adolescência: contribuições para a enfermagem. Cogitare enferm. [Internet] 2012;17(4). [acesso em 05 mar 2013]. Disponível: http://ojs.c3sl.ufpr.br/ojs2/index.php/ cogitare/article/viewFile/30381/19657

9. Sampaio J, Santos RC, Callou JLL, Souza BB. Ele não quer com camisinha e eu quero me prevenir: exposição de adolescentes do sexo feminino às DST/aids no semi-árido nordestino. Saúde soc. [Internet] 2011;20(1) [acesso em 01 mar 2013]. Disponível: http://www.scielo. br/pdf/sausoc/v20n1/19.pdf

10. Texeira CJ. O fenômeno da morte na adolescência sob o olhar de jovens em conflito com a lei [dissertação]. São Paulo (SP): Universidade de São Paulo; 2009.

11. Romero KT, Medeiros EHGR, Vitalle MSS, Wehba J. O conhecimento das adolescentes sobre questões relacionadas ao sexo. Rev. Ass. Med. Bras. [Internet] 2007;53(1) [acesso em 12 jan 2013]. Disponível: http:// www.scielo.br/pdf/ramb/v53n1/12.pdf.
12. Barreto ACM, Santos RS. A vulnerabilidade da adolescente às doenças sexualmente transmissíveis: contribuições para a prática da enfermagem. Esc. Anna Nery. [Internet] 2009;13(4) [acesso em 12 mar 2013]. Disponível: http://www.scielo.br/pdf/ean/v13n4/ v13n4a17.pdf

13. Moura ERF, Gondim PS, Lima DMC, Sousa IO, Evangelista DR. Perfil sexual e reprodutivo e percepção de adolescentes de escola pública sobre comportamento sexual saudável. Rev. APS. [Internet]. 2011;14(1) [acesso em 20 mar 2013] 14(1). Disponível: http://www.aps.ufjf. br/index.php/aps/article/viewArticle/894

14. Dias FLA, Silva KLS, Vieira NFC, Pinheiro PNC, Maia CC. Risco e vulnerabilidades relacionadas a sexualidade na adolescência. Rev. enferm. UERJ. [Internet] 2010;18(3) [acesso em 20 mar 2013]. Disponível: http://www.facenf.uerj.br/v18n3/v18n3a21.pdf

15. Koerich MS, Baggio MA, Backes MST, Backes DS, Carvalho JN, Meirelle BHS, et al. Sexualidade, Doenças Sexualmente Transmissíveis e contracepção: atuação da enfermagem com jovens da periferia. Rev. enferm. UERJ. [Internet] 2010;18(2) [acesso em 20 mar 2013]. Disponível: http://www.facenf.uerj.br/v18n2/v18n2a17.pdf

16. Departamento de Atenção Básica. Brasília-DF: Programa de Saúde na Escola: tecendo caminhos da intersetorialidade. [Internet] [acesso em 20 mar 2013]. Disponível: http://189.28.128.100/dab/docs/geral/folder pse 03.pdf

17. Bretas JRS, Ohara CVS, Jardim DP, Muroya R.L. Conhecimento sobre DST/AIDS por estudantes adolescentes. Rev Esc Enfem USP. [Internet] 2009;43(3) [acesso em 01 mar 2013].Disponível:http://www.scielo. br/pdf/reeusp/v43n3/a08v43n

Cogitare Enferm. 2013 Out/Dez; 18(4):663-8 